\title{
Características clínicas y genético-moleculares de la enfermedad de Huntington en pacientes costarricenses: experiencia de 14 años de diagnóstico molecular
}

\author{
Melissa Vásquez-Cerdas ${ }^{1}$, Fernando Morales-Montero ${ }^{2}$ y Patricia Cuenca-Berger ${ }^{2}$ \\ ${ }^{1}$ Instituto de Investigaciones en Salud (INISA), Universidad de Costa Rica, Costa Rica; ${ }^{2}$ Instituto de Investigaciones en Salud (INISA) y Escuela \\ de Medicina, Universidad de Costa Rica, Costa Rica
}

\begin{abstract}
Resumen
Introducción: La enfermedad de Huntington (HD) es un trastorno neurodegenerativo hereditario, caracterizado por signos y síntomas motores, cognitivos y neuropsiquiátricos, causado por una expansión del trinucleótido citosina-adenina-guanina (CAG) en el gen HTT. Es el trastorno neurológico monogénico más común en los países desarrollados, con una prevalencia de 10.6-13.7/100,000 habitantes (población caucásica). Objetivo: Presentar un resumen descriptivo y actualizado de los resultados de estudios genéticos sobre la HD en Costa Rica realizados desde 2004. Métodos: Se realizó un diagnóstico molecular a pacientes con diagnóstico clínico o sospechoso de HD y a familiares asintomáticos con el $50 \%$ de riesgo. Se les brindó asesoramiento genético. Resultados: En 2004 se logró implementar el diagnóstico molecular de la HD en el Instituto de Investigaciones en Salud (INISA), pues antes de 2004 sólo se hacía diagnóstico clínico. Desde 2004 hasta la fecha, el diagnóstico molecular se ha realizado a 135 personas. De éstas, 56 tenían un diagnóstico clínico o sospechoso de Huntington; sin embargo, sólo se encontró la mutación en 33 de ellas. Las restantes 79 personas corresponden a familiares en riesgo, y de éstas sólo 22 resultaron ser portadoras de la mutación (asintomáticas en el momento de la toma de la muestra). Así, desde que se implementó el diagnóstico molecular hasta la fecha hemos identificado a 55 personas con la mutación: 35 mujeres (64\%) y 20 hombres (36\%). Las características clínicas y genético-moleculares de la HD en pacientes costarricenses son similares a las de otras poblaciones. Conclusiones: El diagnóstico molecular permite prevenir la ocurrencia y recurrencia de esta enfermedad. Además, es de gran ayuda en el diagnóstico diferencial de enfermedades similares. El diagnóstico presintomático cubre las siguientes expectativas de las personas: aliviar la incertidumbre, planear el cuidado de la salud y conocer si los hijos tienen riesgo.
\end{abstract}

Palabras clave: Costa Rica. Enfermedad de Huntington. Diagnóstico molecular. Genética.

\section{Correspondencia:}

Melissa Vásquez-Cerdas

Instituto de Investigaciones en Salud (INISA)

Universidad de Costa Rica

Ciudad Universitaria Rodrigo Facio

Fecha de recepción: 22/01/2018

Disponible en internet: 13-11-2018

C.P. 2060, San José, Costa Rica

Fecha de aceptación: 08/05/2018

Rev Mex Neuroci. 2018;19(5):9-18

E-mail: melissa.vasquez@ucr.ac.cr

DOI: 10.24875/RMN.M18000003

www.revmexneuroci.com

1665-5044/@ 2018. Academia Mexicana de Neurología A.C. Publicado por Permanyer México. Este es un artículo Open Access bajo la licencia CC BY-NC-ND (http://creativecommons.org/licenses/by-nc-nd/4.0/). 


\title{
Clinical, genetic and molecular characteristics of Huntington's disease in Costa Rican patients: experience of 14 years of molecular diagnosis
}

\begin{abstract}
Objective: The aim is to present a descriptive and updated summary of the results of clinical and genetic studies of Huntington's disease in Costa Rica, carried out since 2004. Methods: Molecular diagnosis was carried out in patients with clinical diagnosis or suspect of Huntington's disease, and their asymptomatic relatives at $50 \%$ risk. Patients received genetic counseling. Results: In 2004 the molecular diagnosis of Huntington's disease in Health Research Institute was implemented, because before 2004 only clinical diagnosis was done. From 2004 to date, the molecular diagnosis was made to 135 people. Of these individuals, 56 had a clinical or suspected diagnosis of Huntington. However, the mutation was found only in 33 of these patients (mutation carriers). The remaining 79 people are relatives at risk and of these, only 22 people were found to be carriers of the mutation (asymptomatic at the moment of sampling). Thus, since the molecular diagnosis was implemented, we have identified 55 people with the mutation; 35 women (64\%) and 20 men (36\%). Clinical and molecular genetic characteristics of Costa Rican Huntington's disease patients are similar to other populations. Conclusions: The molecular diagnosis can prevent the occurrence and recurrence of Huntington's disease. There are other diseases similar to Huntington's disease, therefore, molecular diagnosis is a helpful tool in order to establish the proper clinical diagnosis. Accordingly to our experience, presymptomatic testing fully addresses the following individual's expectancies: uncertainty relief, knowledge of the risk of transmitting the disease to their children and health care planning in the coming years.
\end{abstract}

Key words: Costa Rica. Huntington's disease. Molecular Diagnosis. Genetic.

\section{Introducción}

La HD es un trastorno neurodegenerativo de herencia autosómica dominante caracterizado por signos y síntomas motores (corea, distonía, parkinsonismo), cognitivos y neuropsiquiátricos (depresión, irritabilidad, apatía, agresividad). La pérdida neuronal progresiva de células dentro del circuito corteza-estriado-tálamo-corteza, principalmente en el estriado (núcleo caudado y putamen), conduce a esta presentación clínica'.

Es el trastorno neurológico monogénico más común en los países desarrollados, con una prevalencia en la población caucásica (oeste de Europa) de 10.6-13.7 casos por cada 100,000 habitantes $^{2,3}$, aunque algunas poblaciones tienen una frecuencia más alta ${ }^{4}$. La HD es causada por una mutación que consiste en una expansión inestable del trinucleótido CAG, el cual está localizado cerca del extremo 5' en el exón 1 del gen HTT, ubicado en el cromosoma $4(4 p 16.3)^{5}$. La repetición es polimórfica en la población general, presentando entre 6 y 35 CAG, mientras que los individuos afectados de HD presentan 40 o más repeticiones $\mathrm{CAG}^{6}$.

Desde que en 1983 se localizó el gen asociado a la $\mathrm{HD}$ en el cromosoma $44^{7}$, se iniciaron los protocolos para el diagnóstico molecular por estudios de ligamiento; pero no fue hasta que se descubrió la mutación, en $1993^{5}$, que se pudo hacer un diagnóstico molecular preciso. El curso de la enfermedad es progresivo e incurable, y dado que por el momento los tratamientos se dirigen a reducir la sintomatología, el diagnóstico molecular y el respectivo asesoramiento genético son sumamente importantes. En Costa Rica, los servicios de neurología de los hospitales nacionales no realizan este tipo de estudios moleculares, por lo cual remiten muchos de los casos al INISA. El objetivo de este manuscrito es presentar un resumen descriptivo y actualizado de los resultados de los estudios genéticos sobre la HD en Costa Rica realizados desde el año 2004.

\section{Métodos}

Estudio original, descriptivo y actualizado de los resultados de los estudios genéticos sobre la HD en Costa Rica.

\section{Población de estudio}

Sujetos costarricenses con un diagnóstico clínico o sospechoso de HD referidos por un neurólogo, además de familiares con riesgo de presentar la mutación que causa la HD.

A cada individuo con diagnóstico clínico o sospechoso de HD, así como a sus familiares, se les explicó al detalle las características clínicas y genéticas de la enfermedad. Se les informó sobre el patrón de herencia, sus niveles de riesgo, el significado del estudio genético y finalmente se les consultó sobre la necesidad e interés de contar con los beneficios que ofrece el diagnóstico molecular de la HD, tanto el diagnóstico confirmatorio (para pacientes con diagnóstico clínico de la HD) como 
el presintomático (para individuos asintomáticos con el $50 \%$ de riesgo de padecer la $\mathrm{HD}$ ). Las personas que aceptaron realizarse el diagnóstico molecular firmaron la fórmula de consentimiento informado aprobada por el Comité Ético Científico de la Universidad de Costa Rica.

\section{Diagnóstico molecular}

\section{EXTRACCIÓN DE ADN}

Una vez firmada la fórmula de consentimiento, a cada sujeto se le tomó una muestra de sangre periférica, de donde se obtuvo el ADN a partir de leucocitos mediante extracción con fenol-cloroformo, según los procedimientos habituales ${ }^{8}$.

\section{REACCIÓN EN CADENA DE LA POLIMERASA Y ELECTROFORESIS}

Inicialmente, para determinar el número de las repeticiones CAG se realizaba la reacción en cadena de la polimerasa (PCR) utilizando el protocolo descrito por Warner, et al. ${ }^{9}$, con algunas modificaciones, usando los iniciadores HD1 y HD3, que amplifican selectivamente las repeticiones CAG. Luego, los productos de la PCR se sometían a una electroforesis sobre geles de poliacrilamida desnaturalizante al $6 \%$ en buffer TBE $1 \mathrm{X} \mathrm{pH}$ de 8.0 por un periodo de $2 \mathrm{~h}$ y $50 \mathrm{~min}$, para determinar el tamaño absoluto de los fragmentos. Los fragmentos eran visualizados mediante el método de tinción con nitrato de plata. A partir de 2013 se implementó un nuevo protocolo para determinar el número de repeticiones CAG usando el protocolo de PCR descrito por Liu, et al., ${ }^{10}$ también con algunas modificaciones.

\section{Purificación y secuenciación del ADN}

Tras seguir el protocolo de Liu, et al. $.^{10}, 20$ ul de los productos de la PCR se corrieron en geles de agarosa (Top visión, Fermentas) al 2\%, teñidos con gel red (Fermentas), en buffer TBE $0.5 \mathrm{X} \mathrm{pH}$ de 7.7 durante $3 \mathrm{~h}$ a $100 \mathrm{~V}$. Los productos de la PCR fueron extraídos del gel y purificados utilizando el kit QIAquick Gel Extraction (QIAGEN). Posteriormente, los productos purificados se secuenciaron usando el kit Big Dye V3.1 (Applied Biosystems) en un secuenciador automático ABI PRISM 377 (Applied Biosystems). Las secuencias fueron analizadas con el programa 4Peaks (mekentosj.com) con el fin de determinar el número exacto de repeticiones $C A G$.

\section{Asesoramiento y elaboración de material informativo y educativo}

A todos los pacientes y familiares asintomáticos se les brindó asesoramiento genético. Se les habló del significado del estudio genético, la causa de la enfermedad, los síntomas clásicos y su progresión, el patrón de herencia, los riesgos reproductivos, los intervalos de repeticiones y su significado, etc. También se elaboraron y entregaron panfletos con información sobre diferentes temas relacionados con la HD, como nutrición, cuidado dental, fisioterapia, cuidados del paciente con HD, consejos para los cuidadores de pacientes, entre otros.

\section{Resultados}

En 2002 se comenzaron a realizar los primeros experimentos en el INISA para la implementación y aplicación del diagnóstico molecular de la HD en Costa Rica; y ya para el año 2004 se logró optimizar el diagnóstico molecular de la HD. Desde 2004 hasta el día de hoy, el diagnóstico molecular se ha realizado a 135 personas. De éstas, 56 tenían un diagnóstico clínico o sospechoso de HD; sin embargo, la mutación HD se identificó únicamente en 33 pacientes (portadores de la mutación). Las restantes 79 personas corresponden a familiares en riesgo, y de éstas sólo 22 resultaron ser positivas (portadoras de la mutación), aunque asintomáticas en el momento del estudio. Por lo tanto, desde que se implementó el diagnóstico molecular hasta la fecha hemos identificado a 55 personas con la mutación HD: 35 mujeres (64\%) y 20 hombres (36\%). Los pacientes con la HD se encuentran distribuidos a lo largo de todo el territorio nacional, concentrándose la mayoría de ellos en el Valle Central. En tres de las familias se concentra el mayor número de afectados (27 de 55). Las características moleculares de estos 55 individuos positivos para la mutación se pueden observar en la tabla 1.

Las características clínicas y genético-moleculares de la HD en pacientes costarricenses son similares a las de otras poblaciones. Todas las personas en las que se encontró la mutación resultaron ser heterocigotas, con un alelo normal y un alelo expandido. El número de repeticiones CAG en los alelos mutados se encuentra entre 40 y $55 \mathrm{CAG}$, excepto en un caso, en el cual se encontró que portaba 125 repeticiones CAG. Los alelos mutados tienen en promedio 46.5 repeticiones 
Rev Mex Neuroci. 2018;19

Tabla 1. Características moleculares de 55 individuos costarricenses con la mutación HD

\begin{tabular}{|c|c|c|c|c|c|}
\hline $\begin{array}{l}\text { Código del } \\
\text { individuo }\end{array}$ & Sexo & Transmisión & $\begin{array}{c}\text { Edad de } \\
\text { inicio (años) }\end{array}$ & $\begin{array}{c}\text { Número de CAG alelo } \\
\text { normal }\end{array}$ & $\begin{array}{c}\text { Número de CAG alelo } \\
\text { expandido }\end{array}$ \\
\hline HD1 & $F$ & Paterna & 40 & 17 & 46 \\
\hline HD2 & $\mathrm{M}$ & Materna & 39 & 27 & 46 \\
\hline HD3 & $F$ & Materna & Asintomática & 28 & 49 \\
\hline HD4 & $F$ & Materna & Asintomática & 28 & 46 \\
\hline HD5 & $\mathrm{M}$ & Paterna & 30 & 16 & 50 \\
\hline HD6 & $\mathrm{M}$ & Paterna & 30 & 26 & 48 \\
\hline HD13 & $\mathrm{F}$ & Paterna & 20 & 19 & 55 \\
\hline HD19 & $\mathrm{F}$ & Materna & 41 & 14 & 47 \\
\hline HD22 & $F$ & Materna & Asintomática & 17 & 43 \\
\hline HD23 & $\mathrm{M}$ & Paterna & 25 & 15 & 55 \\
\hline HD26 & M & Materna & 53 & 17 & 42 \\
\hline HD27 & $\mathrm{M}$ & Paterna & Asintomático & 17 & 42 \\
\hline HD28 & $\mathrm{M}$ & Materna & 53 & 26 & 42 \\
\hline HD29 & $F$ & Paterna & Asintomática & 17 & 47 \\
\hline HD30 & $\mathrm{F}$ & Materna & Asintomática & 17 & 43 \\
\hline HD34 & $F$ & Paterna & 28 & 26 & 47 \\
\hline HD37 & M & Paterna & 40 & 28 & 46 \\
\hline HD38 & $F$ & Paterna & 40 & 17 & 46 \\
\hline HD42 & $F$ & Paterna & 37 & 17 & 53 \\
\hline HD47 & $\mathrm{M}$ & Materna & 65 & 24 & 44 \\
\hline HD55 & $F$ & Paterna & 57 & 19 & 44 \\
\hline HD58 & $\mathrm{M}$ & Materna & Asintomático & 15 & 43 \\
\hline HD59 & $\mathrm{F}$ & Paterna & 45 & 19 & 43 \\
\hline HD62 & $\mathrm{M}$ & Materna & Asintomático & 26 & 42 \\
\hline HD68 & $F$ & Materna & 46 & 17 & 44 \\
\hline HD71 & $F$ & Paterna & 40 & 17 & 43 \\
\hline HD73 & $F$ & Materna & 40 & 21 & 41 \\
\hline HD76 & $\mathrm{M}$ & Paterna & 32 & 18 & 42 \\
\hline HD79 & $\mathrm{M}$ & Paterna & 40 & 21 & 45 \\
\hline HD87 & $F$ & Materna & Asintomática & 18 & 40 \\
\hline HD93 & $\mathrm{F}$ & Desconocida & 65 & 17 & 40 \\
\hline HD94 & $\mathrm{M}$ & Paterna & 40 & 17 & 41 \\
\hline HD95 & $\mathrm{F}$ & Paterna & Asintomática & 23 & 46 \\
\hline HD96 & $\mathrm{F}$ & Paterna & Asintomática & 17 & 49 \\
\hline HD97 & $\mathrm{M}$ & Materna & Asintomático & 19 & 41 \\
\hline
\end{tabular}


Tabla 1. Características moleculares de 55 individuos costarricenses con la mutación HD (Continuación)

\begin{tabular}{|c|c|c|c|c|c|}
\hline $\begin{array}{l}\text { Código del } \\
\text { individuo }\end{array}$ & Sexo & Transmisión & $\begin{array}{c}\text { Edad de } \\
\text { inicio (años) }\end{array}$ & $\begin{array}{c}\text { Número de CAG alelo } \\
\text { normal }\end{array}$ & $\begin{array}{c}\text { Número de CAG alelo } \\
\text { expandido }\end{array}$ \\
\hline HD102 & $\mathrm{F}$ & Paterna & 45 & 30 & 45 \\
\hline HD103 & $\mathrm{F}$ & Materna & 49 & 17 & 47 \\
\hline HD104 & $\mathrm{M}$ & Materna & 43.5 & 17 & 46 \\
\hline HD107 & $\mathrm{F}$ & Paterna & 7 & 27 & 125 \\
\hline HD108 & $\mathrm{M}$ & Paterna & Asintomático & 19 & 41 \\
\hline HD109 & $F$ & Paterna & Asintomática & 19 & 40 \\
\hline HD110 & $\mathrm{F}$ & Paterna & Asintomática & 19 & 40 \\
\hline HD114 & $\mathrm{M}$ & Paterna & 18 & 21 & 55 \\
\hline HD118 & $\mathrm{M}$ & Paterna & Asintomático & 17 & 45 \\
\hline HD120 & $\mathrm{F}$ & Paterna & 35 & 17 & 44 \\
\hline HD123 & $F$ & Materna & 32 & 21 & 45 \\
\hline HD124 & $\mathrm{M}$ & Materna & 23 & 18 & 43 \\
\hline HD125 & $\mathrm{F}$ & Paterna & 33 & 18 & 48 \\
\hline HD126 & $\mathrm{F}$ & Materna & 48 & 17 & 43 \\
\hline HD129 & $\mathrm{F}$ & Desconocida & 43 & 22 & 46 \\
\hline HD131 & $\mathrm{F}$ & Paterna & 35 & 18 & 44 \\
\hline HD132 & $\mathrm{F}$ & Materna & 28 & 17 & 55 \\
\hline HD133 & $\mathrm{F}$ & Desconocida & 58 & 17 & 44 \\
\hline HD134 & $\mathrm{F}$ & Desconocida & 59 & 18 & 42 \\
\hline HD138 & $\mathrm{F}$ & Paterna & 39 & 17 & 41 \\
\hline
\end{tabular}

F: Femenino; M: Masculino

CAG (stantard desviation [SD] \pm 19.7 ), siendo el alelo más común el de 46 repeticiones CAG (14.5\%). Los alelos normales más frecuentes presentan 17 (38.2\%) y 19 repeticiones CAG (12.7\%) (Figs. 1 y 2$)$.

Para los pacientes sintomáticos (39 en total), la edad promedio de inicio de la enfermedad fue de $39.4 \pm 12.7$ años (entre 7 y 65 años). La mayoría de los individuos manifestaron 0 desarrollaron la enfermedad entre los 21 y 50 años, principalmente en la cuarta y quinta década de vida (Tablas 1 y 2). Hay tres casos de HD infanto-juvenil $(\leq 20)$ en los que la transmisión fue por vía paterna y estuvieron asociados con tamaños de repeticiones grandes (Tablas 1 y 2 ).

La mutación fue inestable en el $69 \%$ de las transmisiones intergeneracionales (11/16) para las que se tienen datos. En seis de las transmisiones intergeneracionales (cuatro por vía paterna y dos por vía materna) un progenitor afectado heredó a su hijo(a) un alelo mutado expandido, mientras que en las restantes cinco transmisiones (todas por vía materna) se heredó al hijo(a) un alelo con una contracción. En las otras cinco transmisiones, el tamaño de la mutación transmitida al hijo fue del mismo tamaño que tenía el progenitor. En general, los cambios son pequeños, de sólo unas pocas repeticiones CAG, a excepción de un caso (Tabla 3). Durante estos años en los que se ha realizado el diagnóstico molecular en el INISA han surgido algunos casos importantes de mencionar:

- Caso infantil. Quizás es el más importante de destacar, pues es el primer caso de HD infantil en Costa Rica, confirmado mediante análisis genético-molecular en el INISA, referido por el Hospital Nacional de Niños de Costa Rica y que publicamos en $2016^{11}$. Se trata de una niña de siete años de edad que presentaba retraso global en el desarrollo psicomotor, problemas de lenguaje, problemas de aprendizaje, 
Tabla 2. Distribución de los individuos afectados según la edad de inicio y la forma de presentación de la HD

\begin{tabular}{|l|c|l|}
\hline Edad de inicio (años) & Número de individuos afectados (\%) & Forma de presentación (según edad de inicio) \\
\hline$\leq 20$ & $3(8)^{*}$ & HD infantil/juvenil \\
\hline $21-50$ & $29(74)^{\dagger}$ & HD clásica \\
\hline$>50$ & $7(18)$ & HD de inicio tardío \\
\hline
\end{tabular}

* $1 / 3$ pacientes $\leq 10$ años.

${ }^{\dagger} 23 / 29$ pacientes $(79 \%)$ la manifestaron entre los 31 y 50 años.

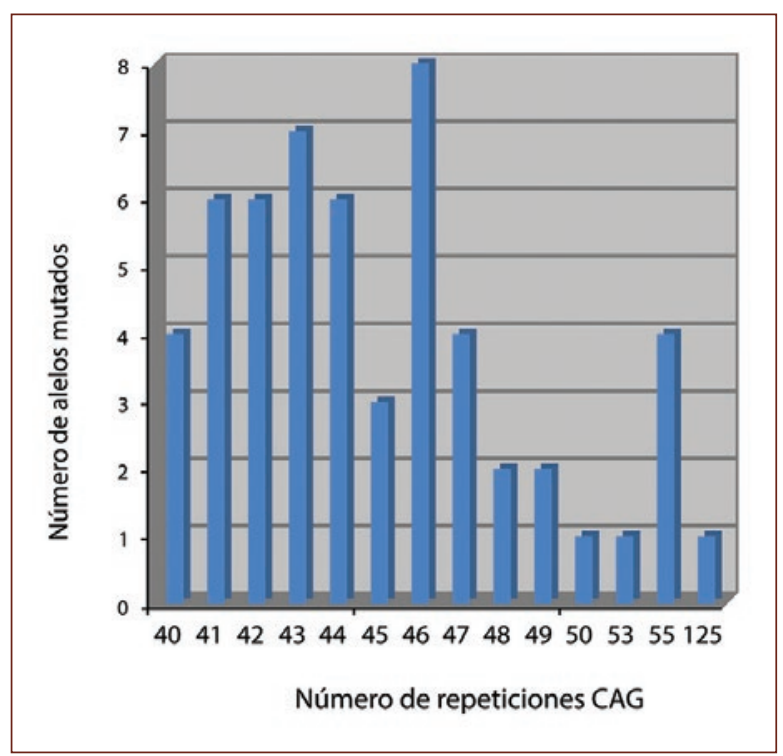

Figura 1. Distribución de repeticiones CAG en alelos mutados de individuos afectados con la HD.

síndrome rígido-acinético y epilepsia. La resonancia magnética documentó atrofia difusa corticosubcortical, del cerebelo y ganglios de la base (caudado y putamen). Los análisis moleculares revelaron que la niña presentaba un alelo mutado de 125 repeticiones CAG, heredado de su padre, quien presentaba un alelo con 55 repeticiones $C A G$ y presentó síntomas alrededor de a los 20 años de edad.

- Caso de inicio tardío (forma senil). Otro caso poco común y completamente opuesto al anterior es el de una mujer de 71 años valorada por cuadro coreico que inició a la edad de 65 años. Según indica el médico tratante (A. Parajeles, de la Clínica Bíblica) mediante comunicación personal, para los familiares lo más evidente era una sensación de «inquietud» en ambas piernas, aunque de predominio izquierdo, con lateropulsión de la marcha asociados a movimientos faciales tipo «tics». Los síntomas progresaron con caída fácil de objetos de las manos. En la primera

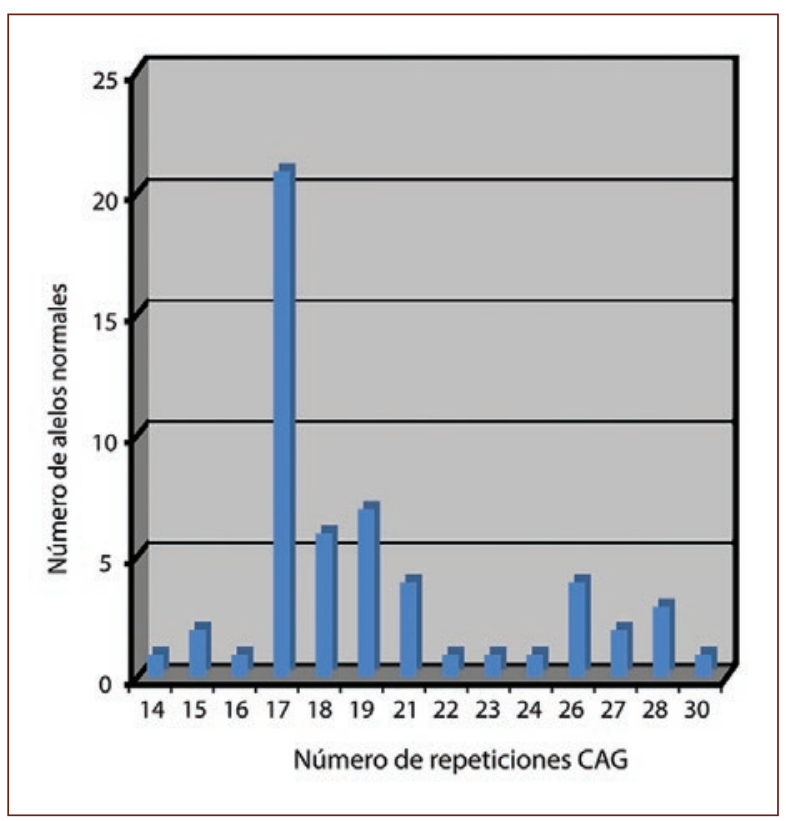

Figura 2. Distribución de repeticiones $C A G$ en alelos normales de individuos afectados con la HD.

valoración neurológica, a los dos años de haberse iniciado los síntomas, eran evidentes los movimientos coreicos de las extremidades inferiores, en especial cuando se dejaban las piernas «suspendidas» en el borde de la camilla; además, presentaba hipotonía leve. Los movimientos no se exacerbaban con el ruido, y la marcha de puntillas y talones estaba conservada. No se tiene información con respecto a los cambios cognitivos. La paciente negó historia familiar de cuadros similares. La TC mostró discreta ventriculomegalia. No se enfatizó acerca del volumen de la cabeza del núcleo caudado. Debido al cuadro clínico que presentaba la paciente, en ese momento se recomendaron estudios genéticos para HD. El diagnóstico molecular indicó que la paciente posee un alelo de 17 y otro de 40 CAG. En la cita de control se observó respuesta parcial a los neurolépticos. Los movimientos de elevación de hombros y de ambas piernas se acentuaban al sentirse observada. El 
Tabla 3. Relación del tamaño de la mutación entre progenitores e hijos(as)

\begin{tabular}{|c|c|c|c|}
\hline $\begin{array}{l}\text { Tamaño de la mutación en el } \\
\text { progenitor }\end{array}$ & Tamaño de la mutación en el hijo(a) & $\begin{array}{l}\text { Cambio intergeneracional } \\
\text { (Número de CAG) }\end{array}$ & Transmisión \\
\hline 46 CAG & $46 \mathrm{CAG}$ & 0 & Materna \\
\hline 46 CAG & 49 CAG & +3 & Materna \\
\hline 46 CAG & 46 CAG & 0 & Materna \\
\hline 47 CAG & $43 \mathrm{CAG}$ & -4 & Materna \\
\hline $50 \mathrm{CAG}$ & 55 CAG & +5 & Paterna \\
\hline $42 \mathrm{CAG}$ & 42 CAG & 0 & Paterna \\
\hline 42 CAG & 47 CAG & +5 & Paterna \\
\hline $47 \mathrm{CAG}$ & 43 CAG & -4 & Materna \\
\hline $55 \mathrm{CAG}$ & 125 CAG & 70 & Paterna \\
\hline $44 \mathrm{CAG}$ & $42 \mathrm{CAG}$ & -2 & Materna \\
\hline 43 CAG & 43 CAG & 0 & Materna \\
\hline $46 \mathrm{CAG}$ & $40 \mathrm{CAG}$ & -6 & Materna \\
\hline $40 \mathrm{CAG}$ & $41 \mathrm{CAG}$ & +1 & Materna \\
\hline 46 CAG & 46 CAG & 0 & Paterna \\
\hline 46 CAG & 49 CAG & +3 & Paterna \\
\hline 46 CAG & 43 CAG & -3 & Materna \\
\hline
\end{tabular}

cuadro motor ha ido progresando con deterioro cognitivo evidente en la prueba del reloj, trastorno del lenguaje tipo disartria e hiporexia con pérdida de peso secundaria.

- Caso de alelo intermedio (IA). También encontramos el caso de un hombre asintomático que presenta alelos de 17 y 29 repeticiones CAG, es decir, posee un IA (los IA tienen entre 27 y 35 CAG).

\section{Discusión}

Los resultados de los análisis moleculares realizados en estos 14 años muestran una distribución del número de repeticiones CAG dentro de los intervalos previamente reportados en otras poblaciones a nivel mundial12-18. Al igual que a nivel mundial, nosotros también observamos que los alelos normales más comunes poseen 17 y 19 repeticiones CAG. Al ser una enfermedad autosómica dominante, los varones y las mujeres son afectados por igual y el riesgo de transmitir el gen a la descendencia es del $50 \%$.

La edad de inicio de la HD, definida como la manifestación de síntomas motores o neurológicos significantes, es altamente variable. Por lo general se presenta entre los 30 y 50 años de edad (HD clásica), pero puede presentarse tanto en jóvenes como en adultos mayores ${ }^{19}$.

La distribución de los pacientes costarricenses (Tabla 2) coincide con los porcentajes de las cuatro formas de presentación de la HD que se han descrito, las cuales dependen de la edad de inicio de los síntomas:

- HD clásica (30-50 años de edad), que representa la mayoría de los casos ( 70\%).

- HD de inicio tardío (> 50 años de edad), que representa aproximadamente el $20 \%$ de los casos.

- HD juvenil (JHD) ( $\leq 20$ años de edad), que representa el $5-10 \%$ de los casos.

- HD infantil (< 10 años de edad), que representa 1-3\% de los casos con HD.

Es importante mencionar que la edad de inicio de la enfermedad se basa en la información provista por el paciente o un familiar cercano ${ }^{20}$. El factor fundamental que influencia la edad de inicio en la $\mathrm{HD}$ es el número de repeticiones CAG. Las expansiones entre 40 y 50 repeticiones CAG son vistas con frecuencia en personas en quienes la enfermedad se presenta entre los 30 y 50 
años (HD clásica), mientras que las formas infanto-juveniles se manifiestan, por lo general, cuando se sobrepasan las 55-60 repeticiones. Existe una fuerte asociación entre la longitud de la repetición CAG y la edad de aparición de la enfermedad; cuantas más repeticiones se tengan, más pronto aparece la enfermedad ${ }^{12,21}$.

Un alelo con expansiones CAG puede expandirse, contraerse o permanecer estable cuando es transmitido de un padre/madre a un hijo(a). Aunque las expansiones y contracciones son comunes en las transmisiones maternas y paternas, se ha visto que en el $70-80 \%$ de los pacientes con JHD, la transmisión es por vía paterna y se asocia con tamaños de repeticiones muy grandes ${ }^{22}$. Así, el sexo del progenitor transmisor es uno de los elementos más importantes que influyen en la estabilidad de los alelos en la HD. Aunque por el momento no se tiene certeza de cuándo ocurre la expansión, se señala a la gametogénesis como la fuente principal de la inestabilidad; sin embargo, se desconoce el mecanismo molecular exacto que causa grandes expansiones durante la gametogénesis en los varones ${ }^{23}$.

La HD es un trastorno raro, y aún más rara en una presentación a edad temprana, como el caso infantil que mencionamos y que reportamos previamente ${ }^{11}$. En adultos, la HD se caracteriza principalmente por movimientos involuntarios (corea), cambios de personalidad y demencia. En los niños, la corea es muy rara como síntoma de presentación, y por el contrario en ellos predomina la bradiquinesia, rigidez de las extremidades y el tronco, disfagia, disartria, distonía y crisis epilépticas. Además, se presenta deterioro cognitivo, retraso del habla y el lenguaje, y son comunes los trastornos o problemas de conducta, que han llevado a diagnósticos erróneos como, por ejemplo, déficit de atención con hiperactividad 20,22,24-28.

En el caso que presentó un inicio tardío de la enfermedad, vemos que, en contraste con el caso infantil, hay un predominio de la clínica coreica, mientras que la función cognitiva se mantuvo relativamente conservada hasta edad avanzada. Es importante recalcar además que el número de repeticiones que presenta la paciente es de $40 \mathrm{CAG}$, el número más bajo de repeticiones dentro del intervalo de alelos HD con penetrancia completa. Según las correlaciones clínicas en la HD, cuanto menor número de repeticiones, más leves son los síntomas y aparecen a edades más tardías. A pesar de que la paciente y sus familiares negaron antecedentes familiares con cuadros similares, fue importante realizar el diagnóstico molecular de HD para dar con el diagnóstico clínico correcto, debido principalmente a que la paciente presentaba una corea senil aparentemente esporádica y se podía haber pensado que los síntomas eran parte del proceso normal de envejecimiento. Hay que recordar que una historia familiar negativa o desconocida no excluye una enfermedad hereditaria como la HD, pues los padres afectados pueden haber fallecido antes de llegar a la edad de inicio de los síntomas o pueden aparecer clínicamente como no afectados debido a fenómenos como penetrancia reducida o a la presencia de un IA.

En cuanto al caso que presentó un IA (29 repeticiones CAG), es importante resaltar que estos casos son poco frecuentes. La prevalencia de IA varía del 1.5 al $5.8 \%$, tanto en la población general como en familias con $\mathrm{HD}^{29}$. La probabilidad de que un IA se expanda dentro del intervalo de penetrancia completa depende de varios factores, que incluyen: el género del padre transmisor, el tamaño del alelo, la configuración molecular de la región alrededor de las repeticiones CAG y su haplotipo. El riesgo puede ser tan alto como del 6-10\% para alelos paternos portando 35 repeticiones $\mathrm{CAG}^{30,31}$. En la actualidad, los IA no se consideran asociados con el fenotipo HD, es decir, se dice que las personas con un IA no desarrollan la enfermedad. Sin embargo, ha ido emergiendo evidencia de que algunos individuos con IA podrían desarrollar manifestaciones neuropatológicas y clínicas, de inicio tardío, similares a las de la HD. Es posible que los IA se presenten como un endofenotipo con potencial de manifestaciones clínicas subsecuentes ${ }^{32,33}$. En relación con esto, también se han encontrado signos clínicos leves en pacientes con IA en otras enfermedades causadas por mutaciones inestables, tales como ataxia espinocerebelosa (SCA) de tipo 2 y síndrome del cromosoma $X$ frágil ${ }^{34,35}$. Por lo tanto, todavía existe controversia sobre las consecuencias clínicas de los IA en la HD y sus implicaciones en el asesoramiento genético.

De todos los casos clínica o patológicamente definidos como HD, muchos resultaron negativos para la expansión CAG, es decir, que no tienen la mutación que causa la HD. Esto refleja que existen cuadros clínicos que presentan trastornos del movimiento y que podrían confundirse con la HD, por lo que en estos casos el diagnóstico molecular es de gran ayuda para establecer un diagnóstico clínico correcto. Estos casos negativos son conocidos como fenotipos similares a la HD (HD like Huntington [HDL]) o fenocopias HD. Hasta la fecha, hay cuatro fenocopias, conocidas como HDL1, HDL2, HDL3 y HDL4. Asimismo, existen otras enfermedades que presentan fenotipos similares a la HD, como la atrofia dentatorubral palidolouisiana (DRPLA) ${ }^{36}$. En 


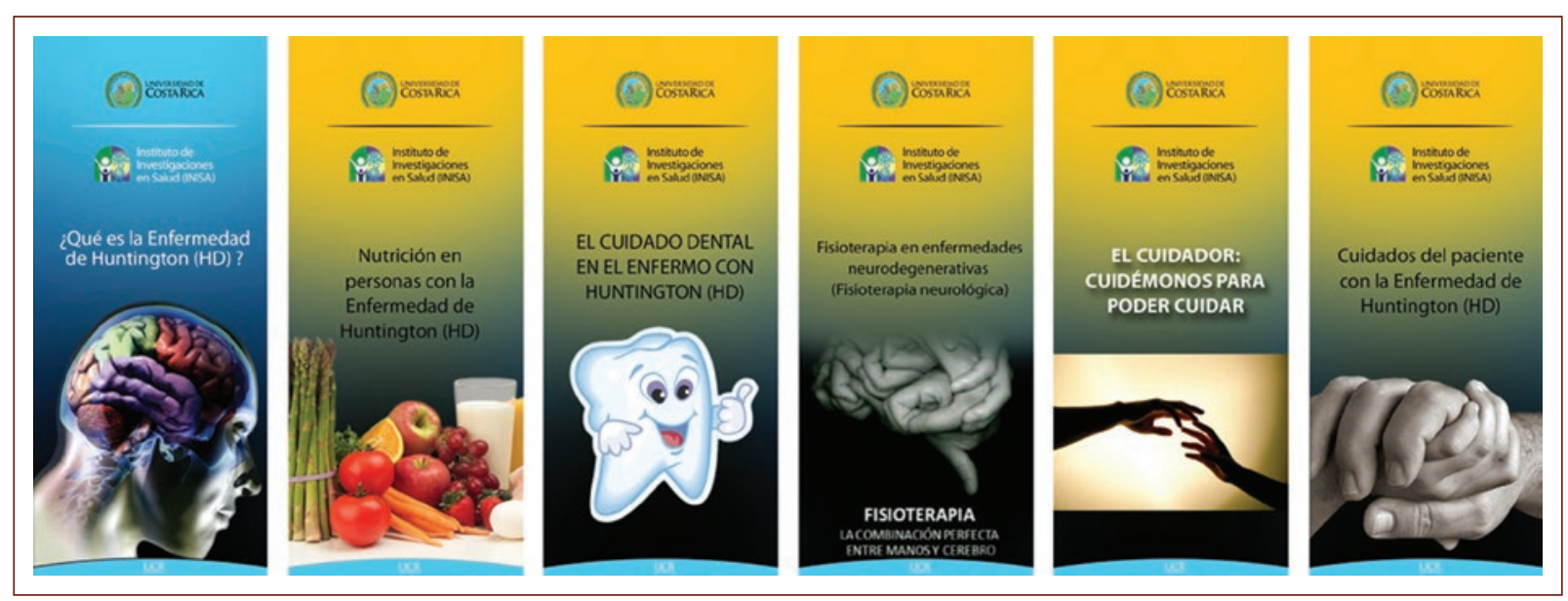

Figura 3. Panfletos informativos y educativos sobre la HD.

nuestro caso, los pacientes sintomáticos que han resultado negativos para la HD, y que han dado su consentimiento, se están tamizando actualmente para las mutaciones que causan la HDL2 y HDL4 (SCA17), que son las fenocopias más comunes, así como para otras enfermedades, como DRPLA, SCA1, SCA2, SCA3 y SCA6, con el fin de contribuir a la correcta clasificación de estos pacientes y optimizar su manejo clínico.

Es importante mencionar que los estudios genéticos de la HD en el INISA ${ }^{37,38}$ a lo largo de los años se han complementado también con el desarrollo de una propuesta de intervención fisioterapéutica en el hogar ${ }^{39}$, pues la terapia física, como parte de un tratamiento interdisciplinario y mediante la optimización de la aptitud física a través del ejercicio, es capaz de mejorar los signos y síntomas, y por ende la funcionalidad, independencia y calidad de vida del paciente con la $\mathrm{HD}^{40}$. Asimismo, la elaboración de material informativo y educativo (Fig. 3) ha sido de mucha ayuda, ya que ha llenado un vacío de información.

\section{Conclusiones}

Hay que tener claro que estos diagnósticos moleculares no pueden predecir cuándo empezarán los síntomas ni cómo será la progresión de la enfermedad. La implementación del diagnóstico molecular de la HD en Costa Rica ha permitido dar a los pacientes y sus familias una clasificación clínica correcta, así como asesoramiento genético oportuno y adecuado. Esperamos que el diagnóstico molecular de la HD permita a mediano y largo plazo una disminución de la recurrencia de personas afectadas con esta enfermedad en el país.

\section{Declaración de conflictos de interés}

No hay ningún potencial conflicto de interés por parte de los autores.

\section{Fuentes de financiamiento}

A lo largo de los años han colaborado el Consejo Nacional para Investigaciones Científicas y Tecnológicas (CONICIT) de Costa Rica, la Vicerrectoría de Investigación y la Vicerrectoría de Acción Social de la Universidad de Costa Rica.

\section{Agradecimientos y colaboradores}

A William Araya Hidalgo y Dayana Vargas Sanabria, del INISA, por su ayuda técnica. Al CONICIT y a la Vicerrectoría de Investigación y Vicerrectoría de Acción Social de la Universidad de Costa Rica.

\section{Bibliografía}

1. Novak M, Tabrizi S. Huntington's disease: clinical presentation and treatment. Int Rev Neurobiol. 2011;98:297-323.

2. Morrison P, Harding-Lester S, Bradley A. Uptake of Huntington disease predictive testing in a complete population. Clin Genet. 2011; 80(3):281-6.

3. Evans SJ, Douglas I, Rawlins MD, Wexler NS, Tabrizi SJ, Smeeth L. Prevalence of adult Huntington's disease in the UK based on diagnoses recorded in general practice records. J Neurol Neurosurg Psychiatry. 2013;84(10):1156-60.

4. Pringsheim T, Wiltshire K, Day L, Dykeman J, Steeves T, Jette N. The Incidence and Prevalence of Huntington's Disease: A Systematic Review and Meta-analysis. Mov Disorders. 2012;27(9):1083-91.

5. The Huntington's Disease Collaborative Research Group. A novel gene containing a trinucleotide repeat that is expanded and unstable on Huntington's disease chromosomes. The Huntington's Disease Collaborative Research Group. Cell. 1993;72:971-83.

6. Bean L, Bayrak-Toydemir P. American College of Medical Genetics and Genomics Standards and Guidelines for Clinical Genetics Laboratories, 2014 edition: technical standards and guidelines for Huntington disease. Genet Med. 2014;16(12):e2.

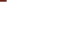


7. Gusella JF, Wexler NS, Conneally PM, Naylor SM, Anderson MA Tanzi RE, et al. A polymorphic DNA marker genetically linked to Huntington's disease. Nature. 1983;306(5940):234-8.

8. Straus WM. Preparation of genomic DNA from mammalian tissue. En: Ausubel FM, R Brent, RE Kinston, eds. Current protocols in molecular biology. New York: Wiley; 1988. 2.2.1-2.2.3.

9. Warner JP, Barron LH, Brock DJH. A new polymerase chain reaction (PCR) assay for the trinucleotide repeat that is unstable and expanded on Huntington's disease chromosome. Mol Cell Probes. 1993;7(3):235-9.

10. Liu $Y$, Shen $Y$, Li H, Wang H, Yang ZR, Chen $Y$, et al. Intergeneration CAG expansion in a Wuhan juvenile-onset Huntington disease family. Neuroscience Bull. 2007;23(4):198-202.

11. Vásquez M, Sevilla F, Gutiérrez A, Cuenca P, Morales F. Enfermedad de Huntington infantil: reporte del primer caso en Costa Rica confirmado por análisis molecular. Neuroje. 2016;29(2):18-25.

12. Andrew SE, Goldberg YP, Kremer B, Telenius H, Theilmann J, Adam S, et al. The relationship between trinucleotide (CAG) repeat length and clinical features of Huntington's disease. Nat Genet. 1993:4(4):398-403.

13. Sánchez A, Castellvi-Bel S, Mila M, Genis D, Calopa M, Jiménez D, et al Huntington's disease: confirmation of diagnosis and presymptomatic testing in spanish families by genetic analysis. J Neurol Neurosurg Psychiatry. 1996;61(6):625-7.

14. Alonso ME, Yescas P, Cisneros B, Martínez C, Silva G, Ochoa A, et al Analysis of the (CAG)n repeat causing Huntington's disease in a Mexican population. Clin Genet. 1997;51:225-30.

15. Lima e Silva T, Guerra H, Bertuzzo C, Lopes I. Molecular diagnosis of Huntington disease in Brazilian patients. Arq Neuropsiquiatr. 2000;58(1):11-7.

16. Saleem Q, Roy S, Murgood U, Saxena R, Verma IC, Anand A, et al. Molecular analysis of Huntington's disease and linked polymorphisms in the Indian population. Acta Neurol Scand. 2003;108(4):281-6.

17. Akbas F, Erginel-Unaltuna N. DNA testing for Huntington disease in the Turkish population. Eur Neurol. 2003;50(1):20-4

18. The U.S.-Venezuela Collaborative Research Project. Venezuelan kindreds reveal that genetic and environmental factors modulate Huntington's disease age of onset. Proc Nat Acad Sci. 2004;101(10):498-503.

19. Ross C, Aylward E, Wild E, Langbehn DR, Long JD, Warner JH, et al. Huntington disease: natural history, biomarkers and prospects for therapeutics. Nat Rev Neurol. 2014;10(4):204-16

20. González-Alegre P, Afifi A. Clinical Characteristics of Childhood-Onset (Juvenile) Huntington Disease: Report of 12 Patientes and Review of the Literature. J Child Neurol. 2006;21(3):223-9.

21. Lee J, Ramos E, Lee JH, Gillis T, Mysore JS, Hayden MR, et al. CAG repeat expansion in Huntington disease determines age at onset in a fully dominant fashion. Neurology. 2012;78(10):690-5.

22. Nance M, Myers R. Juvenile onset Huntington's disease--clinical and research perspectives. Ment Retard Dev Disabil Res Rev. 2001;7(3):153-7.

23. Bates $G$. The molecular genetics of Huntington disease-a history. Nat Rev Genet. 2005;6:766-73.
24. Yoon G, Kramer J, Zanko A, Guzijan M, Lin S, Foster-Barber A, et al. Speech and language delay are early manifestations of juvenile-onset Huntington disease. Neurology. 2006;67(7):1265-7.

25. Ribai P, Nguyen K, Hahn-Barma V, Gourfinkel-An I, Vidailhet M, Legout A et al. Psychiatric and cognitive difficulties as indicators of juvenile Huntington disease onset in 29 patients. Arch Neurol. 2007;64(6):813-9.

26. Quarrell O, Nance M, Nopoulos P, Gourfinkel-An I, Vidailhet M, Legout A, et al. Managing juvenile Huntington's disease. Neurodegener Dis Manag. 2013;3:267-76.

27. Brackenridge CJ. Factors influencing dementia and epilepsy in Huntington's disease of early onset. Acta Neurol Scand. 1980;62(5):305-11.

28. Cloud L, Rosenblatt A, Margolis R, Ross CA, Pillai JA, Corey-Bloom J, et al. Seizures in Juvenile Huntington's Disease: Frequency and Characterization in a Multicenter Cohort. Mov Disorders. 2012;27(14):1797-800.

29. Cubo E, Ramos-Arroyo M, Martínez-Horta S, Martínez-Descalls A, Calvo S, Gil-Polo $\mathrm{C}$, et al Clinical manifestations of intermediate allele carriers in Huntington disease. Neurology. 2016;87(6):571-8.

30. Goldberg YP, McMurray CT, Zeisler J, Almqvist E, Sillence D, Richards F, et al. Increased instability of intermediate alleles in families with sporadic Huntington disease compared to similar sized intermediate alleles in the general population. Hum Mol Genet. 1995;4(10):1911-8.

31. Chong SS, Almqvist E, Telenius H, LaTray L, Nichol K, Bourdelat-Parks B, et al. Contribution of DNA sequence and CAG size to mutation frequencies of intermediate alleles for Huntington disease: evidence from single sperm analyses. Hum Mol Genet. 1997;6(2):301-9.

32. Kenney C, Powell S, Jankovic J. Autopsy-proven Huntington's disease with 29 trinucleotide repeats. Mov Disord. 2007;22(1):127-30.

33. Ha AD, Jankovic J. Exploring the correlates of intermediate CAG repeats in Huntington disease. Postgrad Med. 2011:123(5):116-21.

34. Elden AC, Kim HJ, Hart MP, Chen-Plotkin AS, Johnson BS, Fang X, et al. Ataxin-2 intermediatelength polyglutamine expansions are associated with increased risk for ALS. Nature. 2010;466(7310):1069-75.

35. Hagerman RJ, Hagerman PJ. The fragile $X$ premutation: into the phenotypic fold. Curr Opin Genet Dev. 2002;12(3):278-83.

36. Wild E, Tabrizi S. Huntington's disease phenocopy syndromes. Curr Opin Neurol. 2007;20:681-7.

37. Vásquez M, Morales F, Fernández $\mathrm{H}$, del Valle $\mathrm{G}$, Fornaguera J, Cuenca P. Diagnóstico Molecular de la enfermedad de Huntington (HD) en Costa Rica. Acta Med Costarric. 2008;50(1):35-41.

38. Vásquez M, Morales F, Campos D, Gutiérrez B, Fernández H, Cuenca P. Abordaje integral de pacientes afectados con la enfermedad de Huntington (HD) y sus familiares. Acta Med Costarric. 2011;53(3):136-43.

39. Vásquez M, Suárez JD, Álvarez B, Barboza M. Propuesta de intervención fisioterapéutica en el hogar, para pacientes con la enfermedad de Huntington en Costa Rica. Neuroeje. 2015;28(1):12-23.

40. Vásquez M, Suárez JD. Beneficios de la terapia física en la Enfermedad de Huntington (HD). Neuroeje. 2015;28(1):24-36 